\title{
Optimizing the Extent of Resection and Minimizing the Morbidity in Insular High-Grade Glioma Surgery by High-Field Intraoperative MRI Guidance
}

\author{
Li-feng CHEN ${ }^{1,}$, , Yang YANG ${ }^{2,}$, , Xiao-dong MA ${ }^{1}$, Xin-guang YU1', Qiu-ping GUI ${ }^{3}$, Bai-nan XU1 ${ }^{1}$, Ding-biao ZHOU \\ ${ }^{1}$ The Chinese PLA General Hospital, Department of Neurosurgery, Beijing, China \\ ${ }^{2}$ The Chinese PLA General Hospital, Department of Geriatric Neurology, Beijing, China \\ ${ }^{3}$ The Chinese PLA General Hospital, Department of Pathology, Beijing, China \\ ${ }^{*}$ Drs. CHEN and YANG contributed equally to the manuscript.
}

\section{ABSTRACT}

AIM: The result of surgical resection for insular high-grade glioma (HGG) is disappointing due to the complex anatomy of insula and aggressive growth of HGG. The use of intraoperative magnetic resonance imaging (iMRI) was reported to improve the results of glioma surgery. The authors aimed to evaluate the impact of high-field iMRI and functional neuronavigation on the surgical resection of insular HGG.

MATERIAL and METHODS: Between July 2010 and July 2014, 51 insular HGG patients underwent operations guided by combined high-field iMRI and functional neuronavigation. Twenty-two insular HGG patients underwent conventional neuronavigation operations were assessed as the control group. Preoperative and postoperative tumor volumetric scan analysis, Karnofsky performance score (KPS) and follow-up results were reviewed retrospectively.

RESULTS: Residual tumor was detected by the iMRI in 42 patients, and residual tumor of 37 patients was further resected in the iMRI-assisted group. The median extent of resection (EOR) increased significantly from $79 \%(58 \%-100 \%)$ to $[96 \%(86 \%-$ $100 \%), p<0.001]$. The median EOR of iMRI-assisted group [96\% (86\%-100\%)] was significantly higher than that of conventional neuronavigation group [84\% (69\%-100\%); $p=0.031$. Mean residual tumor volume of iMRl-assisted group [0.6 $\left.(0.0-5.2) \mathrm{cm}^{3}\right]$ was significantly smaller than that of conventional neuronavigation group [3.8 $(0.0-12.1) \mathrm{cm}^{3} ; \mathrm{p}=0.003$ ]. KPS within 3 days after surgery reduced and KPS at 3 months after surgery improved for both groups. KPS of iMRI-assisted group [90(70-100)] was significantly higher than that of control group [80(60-100); $p=0.021]$ at 3 months after surgery. The median progression-free survival (PFS) of iMRI-assisted group [18(9-42) months] was better than that of control group [15(3-32) months; $p=0.010]$. The median overall survival (OS) of iMRI-assisted group [28(14-49) months] was better than that of control group [18 (7-38) months; $p=0.035]$.

CONCLUSION: Combined high-field $\mathrm{iMRI}$ and functional neuronavigation optimize the extent of resection and minimize the morbidity in insular HGG surgery. Aggressive resection of insular HGG is predictive of improved OS and PFS.

KEYWORDS: Intraoperative MRI, Extent of resection, Neuronavigation, Glioma, Surgery 


\section{INTRODUCTION}

nsula is a deep and complex anatomical region. Insular gliomas are not rare, making up more than $25 \%$ of all low grade gliomas (LGGs) and $10 \%$ of all high grade gliomas (HGGs) (7). Treatment options of insular gliomas remain controversial. The proximity of insular gliomas to critical eloquent cortices and tracts affecting motor, sensory, and language functions, as well as the microvasculature around the tumor serving important language and motor systems carry the high risk of surgical treatment (22).

Resecting insular gliomas is a challenging procedure for most neurosurgeons. The cost-benefit balance of insular HGGs surgery is even more unfavorable than in any other tumors due to short life expectancy of the HGG patient and high risk of insular tumor surgery in the past.

Yasargil and other authors (8-10,13,15,24,35,39,42,44,45) have reported resecting some groups of insular gliomas is possible with substantial but acceptable morbidity. In recent years, some surgical techniques including awake surgery, intraoperative monitoring, 5-aminolevulinic acid (5-ALA) and intraoperative magnetic resonance imaging (iMRI)assisted surgery are evolving continuously $(14,17,19,32)$. Although some studies have demonstrated the value of these techniques, their impact on clinical practice remains controversial $(3,11,25)$.

Combined IMRI and functional neuronavigation has been shown to be helpful in achieving maximal tumor resection and minimal postoperative functional impairment in patients with various intra-axial tumors and pituitary adenomas, and glioma resection was frequently reported as an indication for iMRIguided surgery $(1,5,20,26-30)$. However, there are few reports describing the resection of insular HGGs guided by the iMRI system.

The goal of this retrospective study is to evaluate the additional value and superiority of high-field iMRI-guided insular HGG surgery over conventional neuronavigation surgery by comparing the extent of resection (EOR), neurological deficit, Karnofsky performance score (KPS), progression-free survival (PFS) and overall survival (OS).

\section{MATERIAL and METHODS}

\section{Subjects}

During a 4-year period between July 2010 and July 2014, 51 consecutive patients with histologically verified insular primary glioblastoma (GBM, glioma grade IV) or anaplastic astrocytoma (grade III) were treated using combined highfield iMRI and functional neuronavigation in the Department of Neurosurgery of our hospital. Twenty-two insular primary GBM (grade IV) or anaplastic astrocytoma (grade III) patients underwent conventional neuronavigation. All the patients gave informed consent. The study was approved by the Ethics Committee of the Chinese People's Liberation Army (PLA) General Hospital. The clinical information was reviewed by hospital charts, clinic notes, and operative reports.

\section{Evaluation Methods}

Gender, age, the neurological function, KPS, and neuroimaging evaluations of the patients were recorded preoperatively, within 3 days, and then every 3 months after surgery. The tumor volume of pre- and postoperative magnetic resonance (MR) imaging was analyzed by the same neurosurgeon. Manual segmentation was performed with region-of-interest analysis to measure tumor volumes $\left(\mathrm{cm}^{3}\right)$ on the basis of contrastenhancing tissue seen on post-contrast T1-weighted MR images or T2 fluid-attenuated inversion recovery sequence images. The EOR was classified as $<80 \%, 80-89 \%, 90-99 \%$ and $100 \%$. PFS was defined as the time from initial surgery and demonstration of an increase in tumor size on follow-up MR imaging. OS was defined as the time from initial surgery to death.

\section{Image Acquisition}

A dual-room high-field iMRI suite with a scanner of a movable 1.5T magnet (Siemens Espree, Erlangen, Germany) was installed at our hospital in February 2009 (4). Preoperative and intraoperative images were acquired, including 3D T1weighted and postcontrast 3D T1-weighted magnetizationprepared rapid-acquisition gradient echo sequences, T2weighted sequences, 3D T2 fluid-attenuated inversion recovery sequences and diffusion tensor images. The parameters of the scans have been reported in our previous publications $(4,31,40,41,46)$.

\section{Image Processing}

The imaging data was processed by the neuronavigation planning software iPlan 2.6 (BrainLab, Feldkirchen, Germany). Details of the process are the same as our previous reports $(4,31,40,41,46)$. The first step of image processing was conducting format conversion of the data and infusion of the different image sets. 3D reconstruction image of the tumor (40) was obtained by the object creation module of iPlan 2.6. Tumor segmentation was performed based on the postcontrast 3D T1-weighted anatomical dataset or 3D T2 fluid-attenuated inversion recovery sequence images dataset. The outermost rim of postcontrast 3D T1-weighted sequences or 3D T2 fluidattenuated inversion recovery sequence images were defined as the border for segmentation. The tumor was outlined after segmentation was performed on a slice-by-slice basis in the $3 \mathrm{D}$ anatomical data mode. Then the tumor was reconstructed into a 3D object and the computer calculates the volume of the tumor.

The fiber tracking module of iPlan 2.6 based on a tensor deflection algorithm was used to reconstruct the pyramidal tract and the arcuate fasciculus. Seeds were placed on Broca's and Wernicke's areas to reconstruct the arcuate fasciculus. The first region of interest $(\mathrm{ROI})$ and the second $\mathrm{ROI}$ were placed on the subcortical white matter in the precentral gyrus and the cerebral peduncle to reconstruct the motor tract, respectively. The first $\mathrm{ROI}$ and the second ROI were placed on the subcortical white matter in the postcentral gyrus and the cerebral peduncle to reconstruct the sensory tract, respectively. 


\section{Surgical Techniques}

The surgery was performed under general anesthesia. Patients are positioned in a semilateral position with the head turned parallel to the floor. The surgical approaches include the transcortical approach and transsylvian approach. The neuronavigation microscope (Pentero; CarlZeiss, Oberkochen, Germany) could display the contour of the tumor and the functional data during surgery. All the processed image data were integrated for surgical planning and intraoperative neuronavigation (Figure 1A,B). After a conventional frontotemporal craniotomy, cortical language area, arcuate fasciculus, and the pyramidal tract were identified using functional neuronavigation. The transcortical windows above and below the sylvian fissure were created through nonfunctional cortex, taking care to maintain at least a $0.5 \mathrm{~cm}$ margin from any functional site. All resections were continued until eloquent pathways and the lenticulostriate arteries were encountered around the surgical cavity, and then followed according to functional boundaries.

\section{Statistical Methods}

All descriptive and statistical analysis was performed using the Statistical Package for the Social Sciences version 19.0 (SPSS, Chicago, IL, USA). PFS, OS, and the efficacy of two treatment modalities were analyzed using the Kaplan-Meier Method.

We applied 100\%, 90-99, 80-89, and < 80\% EOR cutoffs to maintain consistency across this study for Kaplan-Meier curves plotting the EOR and OS or PFS.

Postoperative neurological complications at 3 days and 3 months after surgery were assessed and compared in the 2 groups. Categorical data was analyzed by Fisher's exact test. Parametric data was analyzed by t-test for unpaired samples. Nonparametric data was analyzed using the Mann-Whitney-U test. Statistical significance was declared at $p<0.05$.
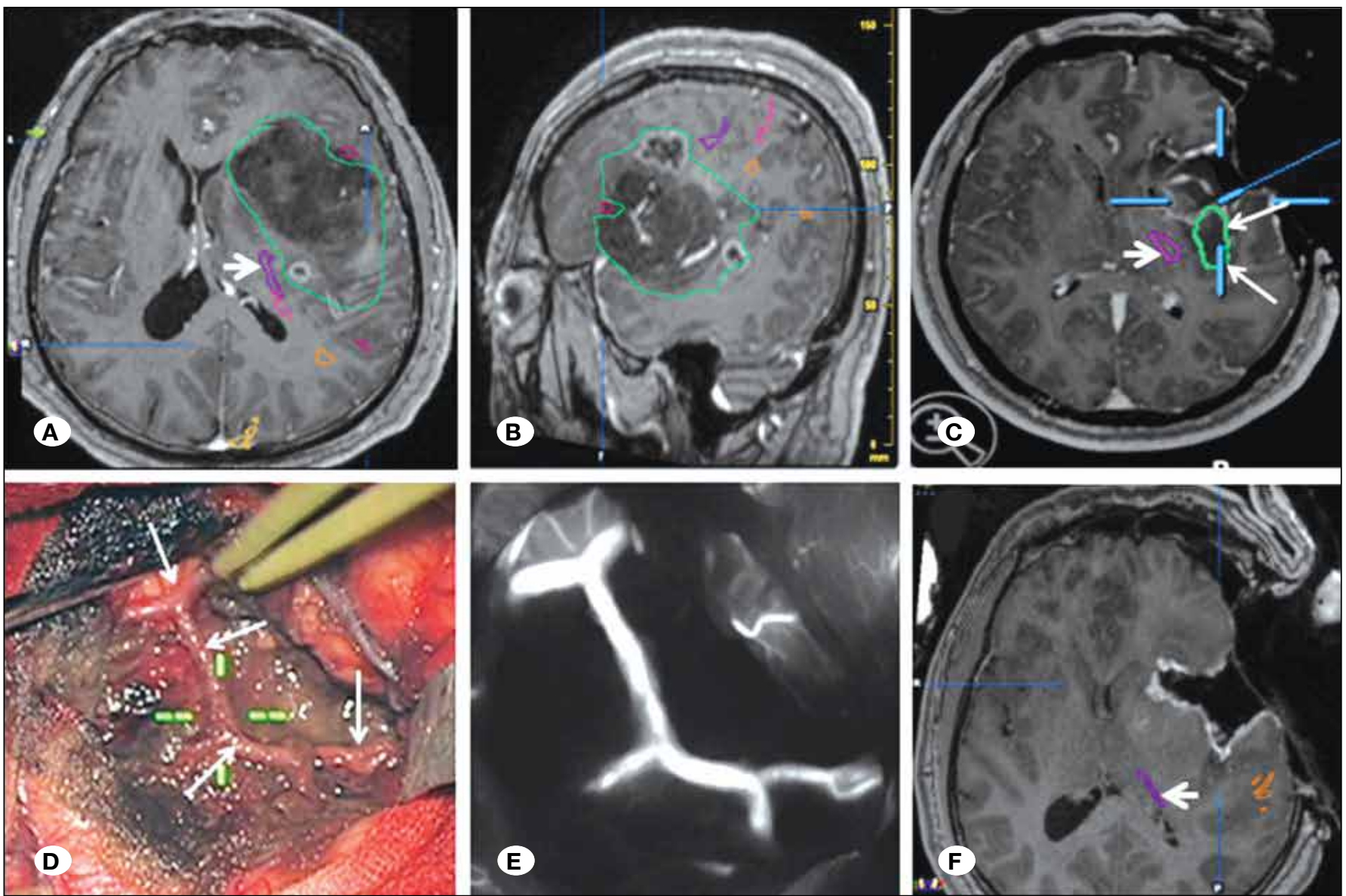

Figure 1: Illustrative case of a 51-year-old man with a giant left insular astrocytoma (grade III-IV), which was successfully removed. Preoperative axial (A) and sagittal (B) T1-weighted contrast-enhanced magnetic resonance imaging (MRI) scan showing a giant left insular glioma. First intraoperative axial T1-weighted contrast-enhanced MRI scan showing a residual mass (thin white arrows, C; 3.2 $\mathrm{cm}^{3}$ ) adjacent to the resection cavity (C). Middle cerebral artery (MCA) and its branches were seen and protected during resection (thin white arrows, D). Intraoperative fluorescent angiography demonstrated MCA and its branches were intact (E). Final intraoperative axial T1-weighted contrast-enhanced MRI scan after additional tumor removal was performed showing complete tumor removal (F). The pyramidal tract was intact during surgery (though white arrows; A, C and F). 


\section{RESULTS}

\section{Preoperative Characteristics}

The patients' demographics, the presenting symptoms, tumor location and volume were shown in Table I. The most common presenting symptom was seizure (50 cases, $68.5 \%)$. Preoperative neuroimaging studies showed glioma hypointense on T1-weighted image and hyperintense on T2weighted image. Sixty-two GBMs showed enhancement on contrast-enhanced MRI in this series (Figure 2A,B).

\section{Intraoperative MRI and Extent of Resection}

The surgical approach, surgical time and postoperative hospital stay are shown in Table I. Residual tumor was detected by the iMRI in 42 patients (Figure 1C), and residual tumor of 37 patients was further resected in the iMRI-assisted group (Figure 1F). The median EOR increased significantly from $79 \%(58 \%-100 \%)$ to $96 \%(86 \%-100 \%)(p<0.001)$ (Figure $3)$. The median EOR of iMRI-assisted group [96\% (86\%$100 \%)$ ] was significantly higher than that of conventional neuronavigation group [84\% (69\%-100\%); $p=0.031]$ (Table I).
Mean residual tumor volume of iMRI-assisted group [0.6 (0.05.2) $\mathrm{cm}^{3}$ ] was significantly smaller than that of conventional neuronavigation group [3.8 (0.0-12.1) $\left.\mathrm{cm}^{3} ; \mathrm{p}=0.003\right]$ (Table I). The major impediment to gross-total resection (GTR) was the difficulty in dissection from the perforating vessels (Figure 1D,E).

\section{Postoperative Course}

There was no perioperative mortality. Table II and Figure 4 show that there is indeed a reduction of KPS at 3 days and improvement at 3 months after surgery for both groups of patients. KPS of iMRI-assisted group 90(70-100) was significantly higher than that of control group [80(60-100); $p=0.021$ ] at 3 months after surgery (Table II, Figure 4). The other surgical outcome at 3 days and 3 months after surgery were summarized in Table II. The senior neuropathologist verified the histological examinations of all the tumors. The histology of tumors according to the World Health Organization (WHO) classification is shown in Table I. All patients received adjuvant conventional radiotherapy and chemotherapy with temozolamide (TMZ) after the initial resection.
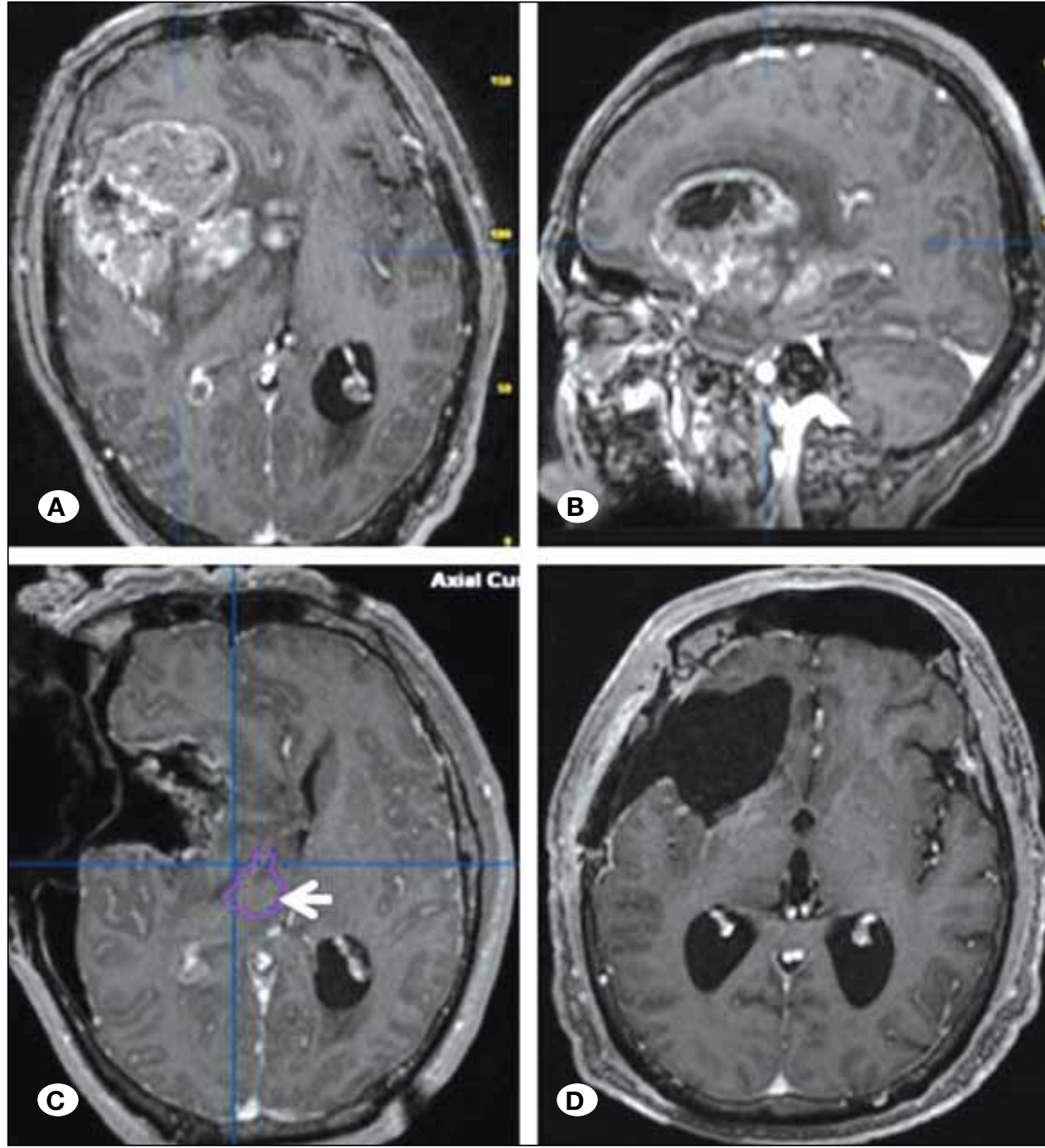

Figure 2: Illustrative case of a 45-year-old woman with a giant right insular glioblastoma (grade IV), which was successfully removed. Preoperative axial (A) and sagittal (B) T1-weighted contrast-enhanced MRI scan showing a giant right insular glioma. Intraoperative axial T1-weighted contrast-enhanced MRI scan showing complete tumor removal and the pyramidal tract was intact during surgery (though white arrow), C). Axial T1-weighted contrast-enhanced MRI scan at 36 months after surgery showing complete tumor removal (D). 


\section{Overall Survival and Progression-Free Survival}

The mean duration of follow-up period is shown in Table I. The re-growth was clinically and radiologically observed in 68 patients $(93.2 \%)$ after the initial resection. Thirty nine patients (53.4\%) underwent re-operation, whereas 12 received adjuvant chemotherapy. Fifty seven patients $(78.1 \%)$ died during the

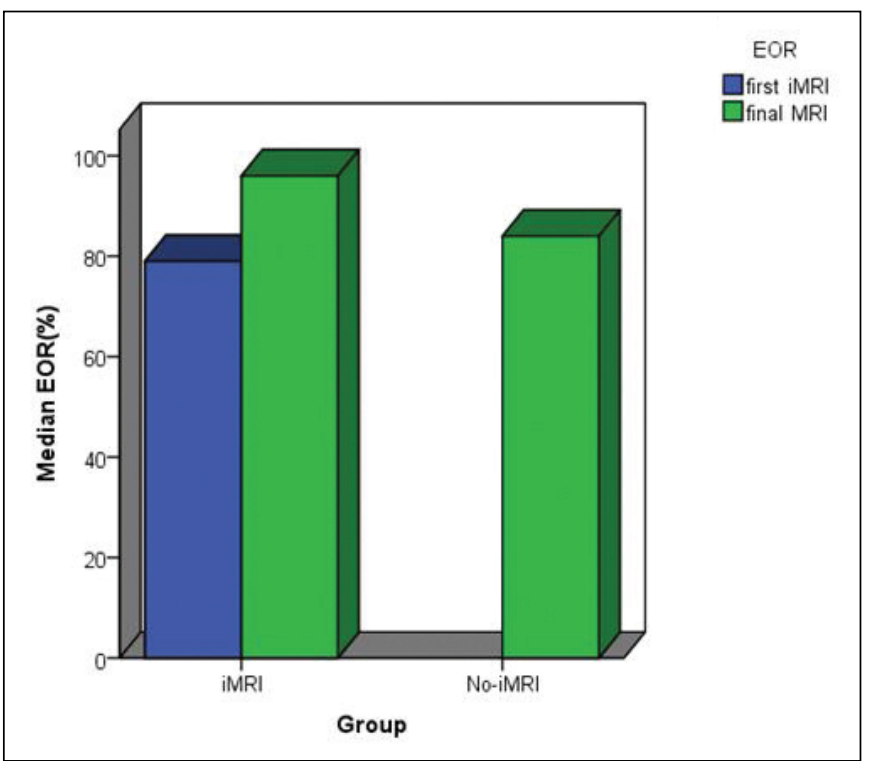

Figure 3: Bar graph showing the extent of resection (EOR, as percentage) in patients undergoing surgery with iMRI guidance and no-iMRI guidance. The percentage of tumor removed with iMRI guidance was determined from the intraoperative initial iMRI (EOR- first iMRI) and from the final MRI (EOR- final iMRI). follow-up period due to tumor progression. The median OS of iMRI-assisted group [28(14-49) months] is better than that of control group [18 (7-38) months; $\mathrm{p}=0.035$ ] (Figure 5A). The median PFS of iMRI-assisted group [18(9-42) months] is better than that of control group [15(3-32) months; $p=0.010$ ] (Figure $5 B)$. Figure $6 \mathrm{~A}, \mathrm{~B}$ shows that aggressive resection of insular HGG is predictive of improved OS and PFS.

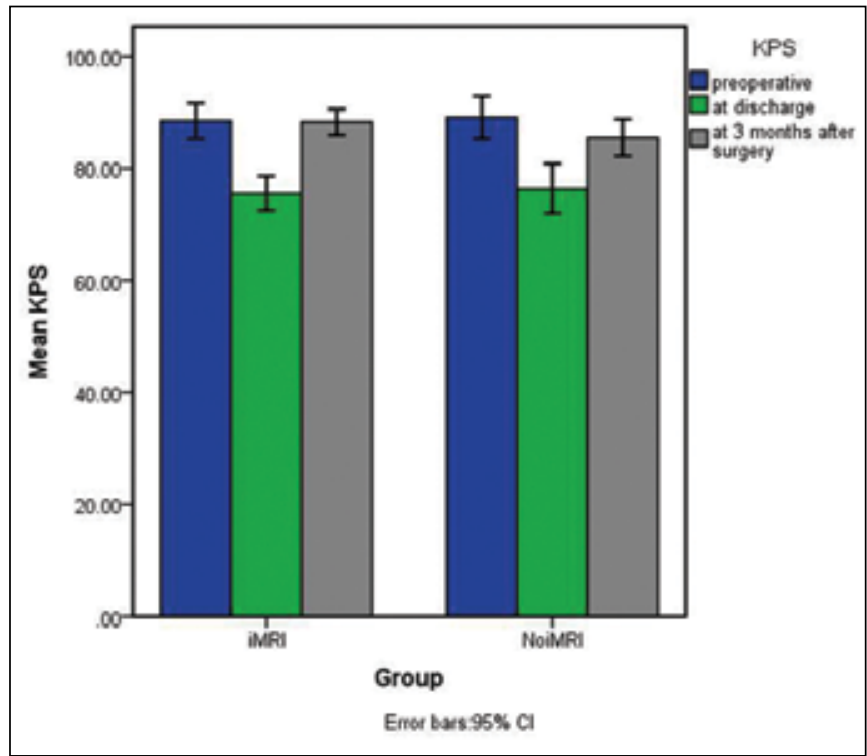

Figure 4: Bar graph showing Karnofsky performance score (KPS) of insular high-grade glioma patients undergoing surgery with iMRI guidance and no-iMRI guidance.
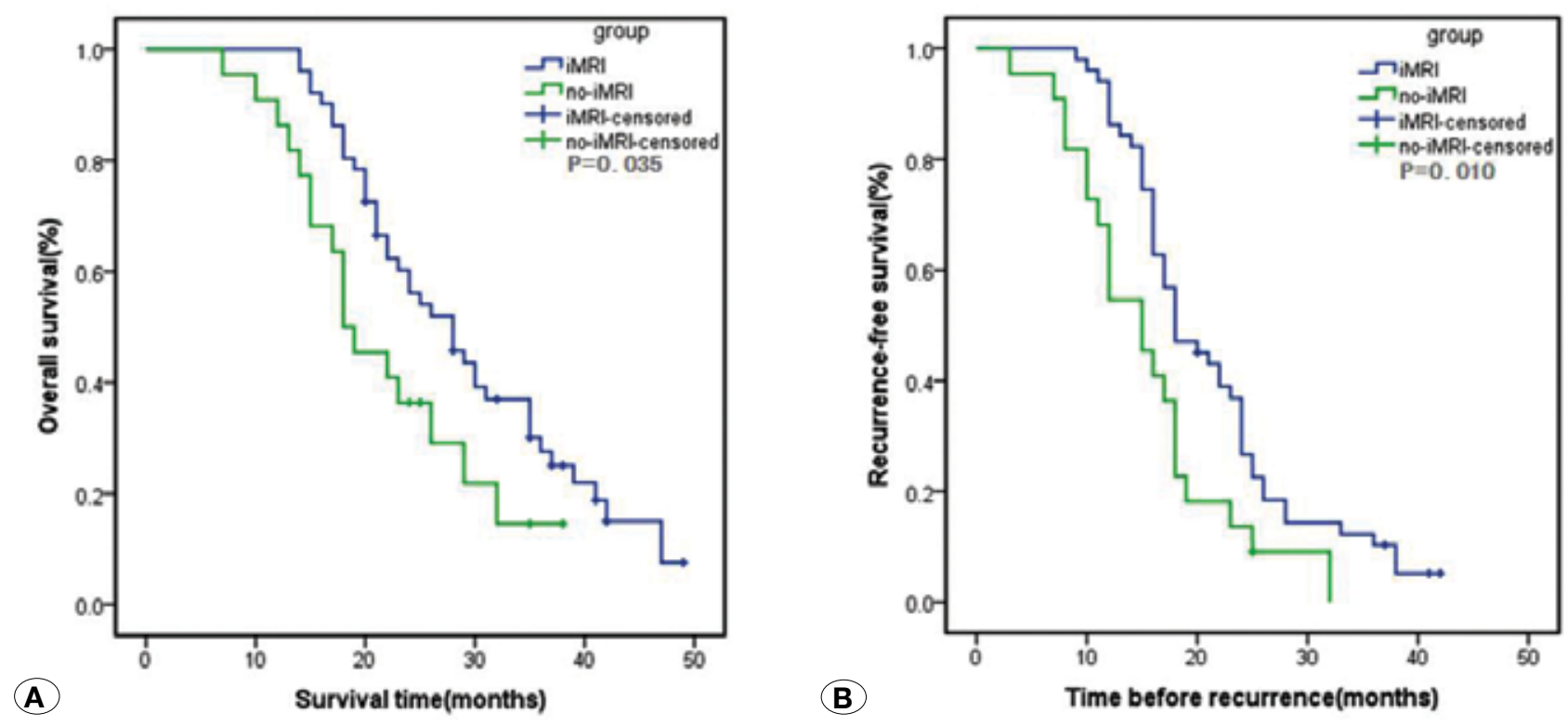

Figure 5: Kaplan-Meier curves showing that combined iMRI and functional neuronavigation operations appeared to extend overall survival (A), and recurrence-free survival (B) compared with conventional neuronavigation operations. 
Table I: Characteristics of 73 Patients Who Underwent Surgical Treatment for Insular HGG from 2010 to 2014

\begin{tabular}{|c|c|c|c|}
\hline Characteristics/ No. of cases & $\begin{array}{c}\text { iMRI-assisted group } \\
(n=51,69.9 \%)\end{array}$ & $\begin{array}{l}\text { No iMRI-assisted group } \\
(\mathrm{n}=22,30.1 \%)\end{array}$ & Results ( $p$ ) \\
\hline Male: Female & $32: 19$ & $14: 8$ & $0.942^{a}$ \\
\hline Mean age (years) & $54.2(23-69)$ & $51.8(25-72)$ & $0.798^{b}$ \\
\hline \multicolumn{4}{|l|}{ Major presenting complaints } \\
\hline Seizure & $36(70.6 \%)$ & $14(63.6 \%)$ & - \\
\hline Headache and dizziness & $18(35.3 \%)$ & $6(27.3 \%)$ & - \\
\hline Motor deficits & $6(11.8 \%)$ & $3(13.6 \%)$ & - \\
\hline Sensory deficits & $4(7.8 \%)$ & $2(9.1 \%)$ & - \\
\hline Language deficit & $3(5.9 \%)$ & $2(9.1 \%)$ & - \\
\hline Incidental & $2(3.9 \%)$ & $1(4.5 \%)$ & - \\
\hline Median pre-operative KPS & $90(50-100)$ & $90(70-100)$ & $0.928^{b}$ \\
\hline Side of tumor in patients (lt/rt) & $21 / 30$ & $9 / 13$ & $0.983^{a}$ \\
\hline \multicolumn{4}{|l|}{ Insular GBM location by zone* } \\
\hline all (giant) & $18(35.3 \%)$ & $7(31.8 \%)$ & - \\
\hline I+IV & $11(21.6 \%)$ & $4(18.2 \%)$ & - \\
\hline $\mathrm{III+IV}$ & $8(15.7 \%)$ & $3(13.6 \%)$ & - \\
\hline $\mathrm{II+III}$ & $7(13.7 \%)$ & $4(18.2 \%)$ & - \\
\hline $\mathrm{I}+\mathrm{II}$ & $4(7.8 \%)$ & $2(9.1 \%)$ & - \\
\hline 1 & $2(3.9 \%)$ & $0(0)$ & - \\
\hline IV & $1(2.0 \%)$ & $2(9.1 \%)$ & - \\
\hline Mean preoperative tumor volume $\left(\mathrm{cm}^{3}\right)$ & $74.5(25.5-180.6)$ & $69.2(21.4-174.0)$ & $0.062^{b}$ \\
\hline Surgical approach (TCO/TSY) & $47 / 4$ & $21 / 1$ & $0.994^{c}$ \\
\hline Surgical time (hours) & $6.5 \pm 0.7$ & $6.3 \pm 0.6$ & $0.716^{d}$ \\
\hline Postoperative hospital stay (days) & $11.5 \pm 2.7$ & $12.1 \pm 3.7$ & $0.892^{d}$ \\
\hline Continued resection after iMRI & $37(72.5 \%)$ & - & - \\
\hline Mean residual tumor volume $\left(\mathrm{cm}^{3}\right)$ & $0.6(0.0-5.2)$ & $3.8(0.0-12.1)$ & $0.003^{b}$ \\
\hline \multicolumn{4}{|l|}{ EOR (extent of resection) } \\
\hline $100 \%$ & $16(31.4 \%)$ & $1(4.5 \%)$ & $0.013^{a}$ \\
\hline $90-99 \%$ & $23(45.1 \%)$ & $7(31.8 \%)$ & $0.357^{a}$ \\
\hline $80-89 \%$ & $12(23.5 \%)$ & $9(40.9 \%)$ & $0.132^{a}$ \\
\hline$<80 \% \#$ & 0 & $5(22.7 \%)$ & - \\
\hline Median & $96 \%(86 \%-100 \%)$ & $84 \%(69 \%-100 \%)$ & $0.031^{b}$ \\
\hline \multicolumn{4}{|l|}{ Tumor histology (WHO grade) } \\
\hline Anaplastic astrocytoma (III) & 7 & 4 & \\
\hline GBM (IV) & 44 & 18 & $0.895^{c}$ \\
\hline
\end{tabular}

TCO: Transcortical, TSY: Transsylvian, KPS: Karnofsky performance score, HGG: High grade glioma, GBM: Glioblastoma, WHO: World Health Organization, iMRI: intraoperative magnetic resonance imaging, It: left, rt: right.

"Berger-Sanai insular glioma classification system (33-35).

"Because of low event numbers, statistical significance was not reached.

aPearson Chi-square Test was used.

${ }^{b}$ Two-Independent-samples Tests (Mann-Whitney) was used.

${ }^{c}$ Continuity Correction Chi-square Test was used.

dIndependent-samples T Test was used. 


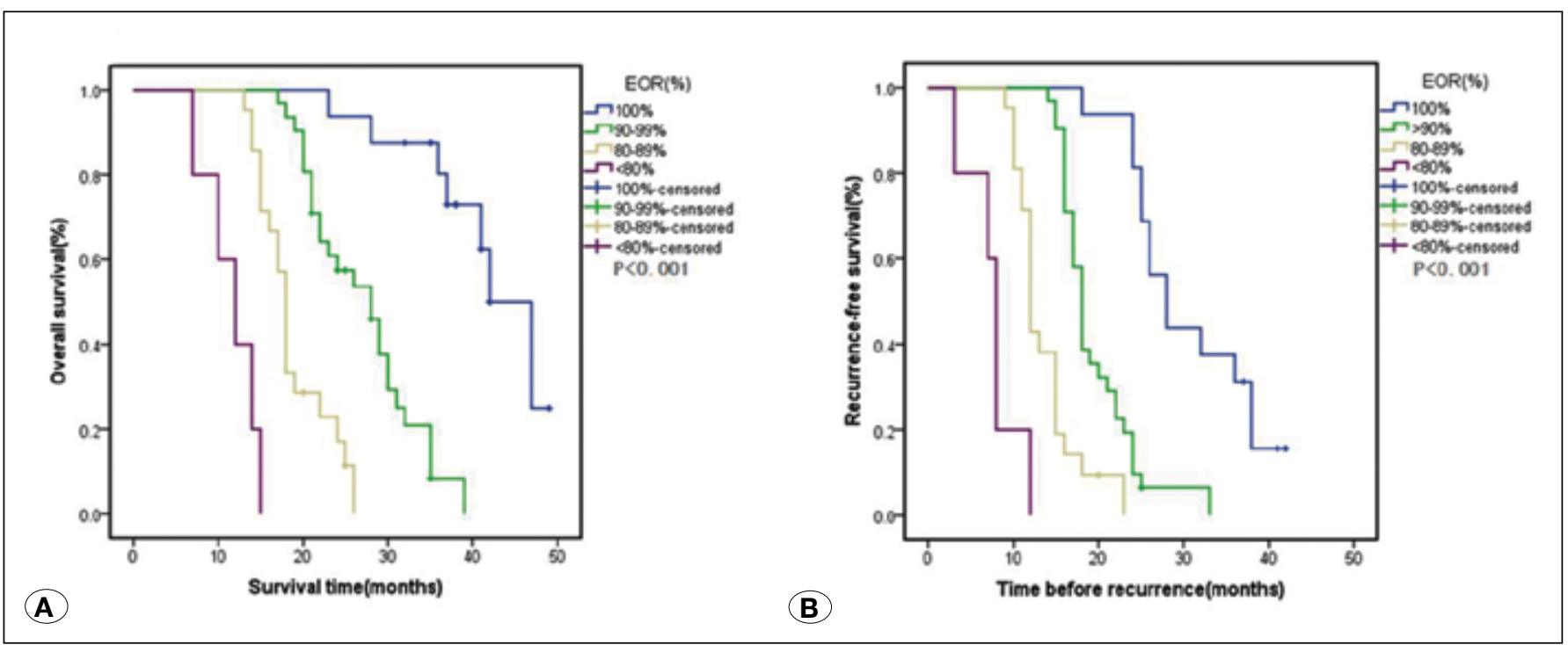

Figure 6: Kaplan-Meier curves revealing the overall survival (A), and recurrence-free survival (B) of patients with insular high-grade glioma, stratified by EOR. More extensive resections may provide overall and recurrence-free survival advantage.

\section{DISCUSSION}

\section{Extent of Resection}

The prognosis of patients with HGG is poor. The goal of glioma surgery is to maximize EOR while minimizing surgical co-morbidities. GTR is desired for the treatment of insular glioma. However, GTR can sometimes be limited by adhesions which the insular glioma may have with surrounding eloquent anatomy and vessels. With improvements in microsurgical techniques, neurological imaging, and electrophysiological monitoring, nervous and vascular structures can be safely identified and manipulated. The complication rates of insular glioma surgery are still significant. The potential benefits of a glioma surgery should be balanced against postoperative neurological complication rates. Significant neurological complication rates of insular HGGs surgery may detract from the patient's quality of life, which will well outweigh any prolongation of survival. So, optimal treatment for insular HGGs remains controversial due to the morbidity inherent to the operation and short life expectancy of the HGG patients.

Although the lack of Class I evidence, data from several studies demonstrated that a maximum safe EOR as a positive predictor for extended OS and PFS for HGG patients $(2,12,23,33,34,36,38,39,43)$. Sanai and Berger (34) also reviewed the major clinical literatures and concluded that more extensive surgical resection is associated with longer life expectancy for patients with glioma. We observed that a better OS and PFS for patients, in whom a 100\% and $90-99 \%$ resection was achieved, but much of the statistical effect was due to the relatively unfavorable prognosis of patients with a $80-89 \%$ and $<80 \%$ tumor resection (Figure 6A,B). Our analysis provides further evidence that greater EOR leads to improved OS and PFS significantly.

Insular HGGs located in the deep region characterized by the lack of anatomical landmarks, paucity of functional information, and risk of overlooking hidden tumor portions. Furthermore, HGGs have infiltrative behavior and intimate co-habitation with brain vascular supply. These factors carry a limitation as regards EOR. Sanai et al.(35) reported the median EOR was $81 \%$ (range $47-100 \%$ ) for insular HGGs. In our series, the median EOR of iMRI-assisted group [96\% (86\%-100\%)] was significantly higher than that of conventional neuronavigation group [84\% (69\%-100\%); $p=0.031]$. Mean residual tumor volume of iMRl-assisted group $\left[0.6 \quad(0.0-5.2) \mathrm{cm}^{3}\right]$ was significantly smaller than that of conventional neuronavigation group [3.8 (0.0-12.1) $\mathrm{cm}^{3} ; \mathrm{p}=0.003$ ]. The better EOR (Table I) in the iMRI-assisted group of our series seemed to be attributed to the combined iMRI and neuronavigation system. Kawaguchi et al.(18) reported that the clear tumor boundaries is one of the indicators for radical resection of insulo-opercular gliomas.

Insular HGGs may invade and compress the surrounding structures, and the borders of the tumor may be difficult to distinguish during surgery. In iMRl-assisted group of our series, iMRI is recommended to update the neuronavigation after brain shift, increase its usefulness for distinguishing tumor borders and avoidance of important structures after resection of the main part of large insular gliomas. The combined iMRI and neuronavigation system is also very helpful when the residual tumor is too small for easy detection. In the iMRIassisted group of our series, residual tumor was detected by the iMRI in 42 patients, and residual tumor of 37 patients was further resected. The median EOR increased significantly from $79 \%(58 \%-100 \%)$ to [96\% (86\%-100\%), p<0.001](Figure 3). If iMRI had not been used to detect the residual tumor, the median EOR would only have been $79 \%$. It showed that iMRI increased the EOR of insular HGG surgery (Figure 2D).

The mid-posterior part of the superior temporal gyrus and the posterior dominant insula are usually both essential for speech function. Furthermore, the posterior insula is very close to the posterior limb of the internal capsule. The posterior part 
Table II: Operative Outcome of 73 Patients Who Underwent Surgical Treatment for Insular HGG

\begin{tabular}{|c|c|c|c|}
\hline Parameters/ Group & $\begin{array}{l}\text { iMRI-assisted } \\
\text { group }(n=51)\end{array}$ & $\begin{array}{l}\text { No iMRI-assisted group } \\
(n=22)\end{array}$ & Results (p) \\
\hline \multicolumn{4}{|l|}{ Hemiparesis } \\
\hline Immediate $^{a}$ & $1(2.0 \%)$ & $4(18.2 \%)$ & $0.044^{c}$ \\
\hline Medium term ${ }^{b}$ & $0(0)$ & $3(13.6 \%)$ & - \\
\hline \multicolumn{4}{|l|}{ Monoparesis } \\
\hline Immediate ${ }^{a}$ & $2(3.9 \%)$ & $4(18.2 \%)$ & $0.116^{c}$ \\
\hline Medium term ${ }^{b}$ & $2(3.9 \%)$ & $3(13.6 \%)$ & $0.316^{c}$ \\
\hline \multicolumn{4}{|l|}{ Facial droop } \\
\hline Immediate $^{a}$ & $2(3.9 \%)$ & $3(13.6 \%)$ & $0.316^{c}$ \\
\hline Medium term $^{b}$ & $0(0)$ & $0(0)$ & - \\
\hline \multicolumn{4}{|l|}{ Sensory deficits } \\
\hline Immediate ${ }^{a}$ & $1(2.0 \%)$ & $3(13.6 \%)$ & $0.147^{c}$ \\
\hline Medium term $^{b}$ & $0(0)$ & $0(0)$ & - \\
\hline \multicolumn{4}{|l|}{ Seizure } \\
\hline Immediate $^{a}$ & $1(2.0 \%)$ & $1(4.5 \%)$ & $1.000^{c}$ \\
\hline Medium term ${ }^{b}$ & $0(0)$ & $0(0)$ & - \\
\hline \multicolumn{4}{|l|}{ Motor aphasia } \\
\hline Immediate $^{a}$ & $0(0)$ & $1(4.5 \%)$ & - \\
\hline Medium term $^{b}$ & 0 & 0 & - \\
\hline \multicolumn{4}{|l|}{ Sensory aphasia } \\
\hline Immediate ${ }^{a}$ & $1(5.9 \%)$ & $4(18.2 \%)$ & $0.044^{c}$ \\
\hline Medium term ${ }^{b}$ & $0(0)$ & $0(0)$ & - \\
\hline \multicolumn{4}{|l|}{ Hematoma } \\
\hline Immediate ${ }^{a}$ & $0(0)$ & $1(4.5 \%)$ & - \\
\hline Medium term ${ }^{b}$ & $0(0)$ & $0(0)$ & - \\
\hline \multicolumn{4}{|l|}{ Infection } \\
\hline Immediate ${ }^{a}$ & $1(3.9 \%)$ & $0(0)$ & - \\
\hline Medium term ${ }^{b}$ & $0(0)$ & $0(0)$ & \\
\hline Postoperative death & $0(0)$ & $0(0)$ & \\
\hline \multicolumn{4}{|l|}{ Total neurological deficits } \\
\hline Immediate ${ }^{a}$ & $8(15.7 \%)$ & $12(54.5 \%)$ & $0.001^{d}$ \\
\hline Medium term ${ }^{b}$ & $2(3.9 \%)$ & $6(27.3 \%)$ & $0.012^{c}$ \\
\hline Median immediate ${ }^{a} \mathrm{KPS}$ & $80(50-90)$ & $80(50-90)$ & $0.683^{e}$ \\
\hline Median medium term ${ }^{b} \mathrm{KPS}$ & $90(70-100)$ & $80(60-100)$ & $0.021^{e}$ \\
\hline Mean follow-up duration, mo & $29.3(14-49)$ & $21.9(7-38)$ & - \\
\hline Median OS, mo & $28(14-49)$ & $18(7-38)$ & 0.035 \\
\hline Median PFS, mo & $18(9-42)$ & $15(3-32)$ & 0.010 \\
\hline
\end{tabular}

OS: overall survival, PFS: progressive-free survival, KPS: Karnofsky performance score, HGG: High grade glioma, mo: months, iMRI: intraoperative magnetic resonance imaging

anew neurological deficits or worsening of pre-existing deficits at discharge.

${ }^{b}$ new neurological deficits or worsening of pre-existing deficits at 3 months after surgery.

'Continuity Correction Chi-square Test was used.

${ }^{d}$ Pearson Chi-square Test was used.

'Two-Independent-samples Tests (Mann-Whitney) was used. 
of the dominant insula limits total removal of insular glioma, and many studies $(6,24,45)$ reported examples of residual glioma in the posterior insular region. To avoid this problem, we usually performed an anterotemporal lobectomy or the superior temporal gyrus lobectomy. Because most of insular HGGs have temporal or frontal extensions, additional cortical resection of superior temporal gyrus maximizes surgical space to provide a better exposure of the residual tumor in the posterior insular region. iMRI and neuronavigation can give the surgeon information about the exact locations of speech function area and the arcuate fasciculus, which help to guide the location of the safe cortical incision in the frontal or temporal gyrus. In iMRI-assisted group of our series, 47 cases were approached through one or more transcortical corridors. Integrating intraoperative functional MRI data and diffusion-tensor imaging-based fiber tracking (DTI-ft) into the neuronavigation system can also help to visualize major white-matter tracts and avoid damage to the pyramidal tract (Figure 1A,C,F and Figure 2C). In iMRI-assisted group of our series, the residual tumor of 3 patients was very close to the pyramidal tract in the posterior limb of the internal capsule, and the surgeon did not continue to resect the residual tumor. The transsylvian approach often requires the sacrifice of some veins, and leads to inferior frontal gyrus and superior temporal gyrus retraction, which may also cause postoperative neurological deficits. In our series, the transsylvian approach was performed in only 5 small volume tumors of early period.

\section{Morbidity}

Aggressive EOR should be weighed against postoperative quality of life, since lower postoperative neurological deficits might be more important with regard to OS than increasing the EOR (21). Postoperative clinical condition of the patient is one of the important prognostic factors, and the patient's quality of life and tumor control should be the ultimate strategies of treatment. Sanai et al.(35) reported the median EOR was $81 \%$ (range $47-100 \%$ ) for insular HGGs, permanent postoperative deficits were noted in 6 patients (6\%). Simon et al.(39) reported a $>90 \%$ resection was achieved in $42 \%$ of cases, KPS scores deteriorated in $41 \%$ of patients at discharge. Our data confirm that in addition to improving the EOR, the combined $\mathrm{iMRI}$ and neuronavigation can increase the safety of tumor resection. We incurred a $96 \%$ median EOR with a permanent postoperative morbidity rate of $2.0 \%$ in the iMRI-assisted group (Table II). Although there was indeed a reduction of KPS at 3 days after surgery, improvement was observed at 3 months after surgery. KPS of iMRI-assisted group [90(70-100)] was significantly higher than that of control group [80(60-100); $p=0.021]$ at 3 months after surgery (Table II, Figure 4). It may imply advantage of the iMRI in enhancing quality of life after resection of insular HGGs.

Duffau (6) reported that almost all severe complications were related to injury of the perforating vessels. Similar to Duffau's report, 2 of 3 patients had postoperative hemiparesis due to injury of perforating vessels in the conventional neuronavigation group of our series.
Intraoperative anatomical alterations and shift of the brain will often cause wrong neuronavigation which is set-up based on preoperative MR imaging. The usual anatomical landmarks and some techniques included intraoperative neurophysiological monitoring and real-time ultrasonography cannot provide the exact location of the perforating arteries to the surgeon. The combined iMRI and neuronavigation can guide the surgeon to identify the location of perforating arteries (Figure $1 \mathrm{D}, \mathrm{E})$. When a HGG encases the important perforating artery, the surgeon should leave a small amount of residual tumor. In iMRI-assisted group of our series, the residual tumor of 2 patients involves the important perforating artery in the anterior perforating substance, and the surgeon left a layer of residual tumor tissue along these vessels in the deep insular region.

\section{Survival and Progression-Free Survival}

The OS and PFS after HGGs surgery depends on many variables, including histological features, age, EOR, and preoperative KPS scores (39). Since the introduction of iMRI, some studies reported that low-field-strength iMRI affected negatively OS of HGG patients. Senft et al.(37) reported 41 GBM patients on a $0.15 \mathrm{~T}$ scanner, and median OS was 88 weeks for the iMRI-group compared with 68 weeks for the conventional neuronavigation group $(p=0.07)$. Hirschberg et al.(16) analyzed 32 patients of GBM on a $0.5 \mathrm{~T}$ iMRI scanner. The result showed mean OS of the iMRI-group and control group without iMRI were 14.5 months and 12.1 months respectively $(p=0.14)$. The high-field iMRI added some benefits compared with low-field iMRI in our study. In our series, 62 of 73 patients had GBMs. No patient has suffered from adverse events during high-field iMRI scanning. Surgical time and postoperative hospital stay time are not different between the iMRI-group and conventional neuronavigation group (Table I). The median PFS of iMRI-assisted group [18(942) months] is better than that of the control group [15(3-32); $\mathrm{p}=0.010]$. The median OS of iMRI assisted group [28(14-49)] is better than that of conventional neuronavigation group [18 (7-38); $p=0.035]$. The OS and PFS in iMRI-assisted group of our series were generally quite good for HGGs patients. Our study demonstrated that high-field iMRI is more effective than conventional neuronavigation-guided surgery in prolonging OS and PFS of insular HGG patients.

\section{- CONCLUSION}

Aggressive resection of insular HGG is predictive of improved $\mathrm{OS}$ and PFS. The techniques of resection guided by combined high-field iMRI and neuronavigation optimize the extent of resection and minimize the morbidity in insular HGG surgery.

\section{ACKNOWLEDGEMENT}

This work was supported by grants from the Sanya innovation fund (no. 2014YW36). 


\section{REFERENCES}

1. Berkmann S, Schlaffer S, Nimsky C, Fahlbusch R, Buchfelder $M$ : Intraoperative high-field MRI for transsphenoidal reoperations of nonfunctioning pituitary adenoma. J Neurosurg 121:1166-1175, 2014

2. Bloch O, Han SJ, Cha S, Sun MZ, Aghi MK, McDermott MW, Berger MS, Parsa AT: Impact of extent of resection for recurrent glioblastoma on overall survival. Clinical article. J Neurosurg 117:1032-1038, 2012

3. Cedzich C, Taniguchi $M$, Schäfer S, Schramm J: Somatosensory evoked potential phase reversal and direct motor cortex stimulation during surgery in and around the central region. Neurosurgery 38:962-970, 1996

4. Chen X, Xu BN, Meng X, Zhang J, Yu X, Zhou D: Dual room $1.5 \mathrm{~T}$ intraoperative magnetic resonance imaging suite with a movable magnet: Implementation and preliminary experience. Neurosurg Rev 35:95-109, 2012

5. Claus EB, Horlacher A, Hsu L, Schwartz RB, Dello-lacono D, Talos F, Jolesz FA, Black PM: Survival rates in patients with low-grade glioma after intraoperative magnetic resonance image guidance. Cancer 103: 1227-1233, 2005

6. Duffau $\mathrm{H}$ : A personal consecutive series of surgically treated 51 cases of insular WHO Grade II glioma: Advances and limitations. J Neurosurg 110:696-708, 2009

7. Duffau H, Capelle L: Preferential brain locations of low-grade gliomas. Cancer 100:2622-2626, 2004

8. Duffau H, Capelle L, Lopes M, Faillot T, Sichez JP, Fohanno D: The insular lobe: Physiopathological and surgical considerations. Neurosurgery 47:801-810, 2000

9. Duffau H, Taillandier L, Gatignol P, Capelle L: The insular lobe and brain plasticity: Lessons from tumor surgery. Clin Neurol Neurosurg 108:543-548, 2006

10. Ebeling U, Kothbauer K: Circumscribed low grade astrocytomas in the dominant opercular and insular region: A pilot study. Acta Neurochir (Wien) 132:66-74, 1995

11. Fadul C, Wood J, Thaler H, Galicich J, Patterson RH Jr, Posner JB: Morbidity and mortality of craniotomy for excision of supratentorial gliomas. Neurology 38:1374-1379, 1988

12. Grabowski MM, Recinos PF, Nowacki AS, Schroeder JL, Angelov L, Barnett GH, Vogelbaum MA: Residual tumor volume versus extent of resection: Predictors of survival after surgery for glioblastoma. J Neurosurg 121:1115-1123, 2014

13. Guenot M, Isnard J, Sindou M: Surgical anatomy of the insula. Adv Tech Stand Neurosurg 29:265-288, 2004

14. Gumprecht H, Ebel GK, Auer DP, Lumenta CB: Neuronavigation and functional MRI for surgery in patients with lesion in eloquent brain areas. Minim Invasive Neurosurg 45:151-153, 2002

15. Hentschel SJ, Lang FF: Surgical resection of intrinsic insular tumors. Neurosurgery 57:176-183, 2005

16. Hirschberg H, Samset E, Hol PK, Tillung T, Lote K: Impact of intraoperative MRI on the surgical results for high-grade gliomas. Minim Invasive Neurosurg 48:77-84, 2005

17. Kamada K, Todo T, Masutani Y, Aoki S, Ino K, Takano T, Kirino T, Kawahara N, Morita A: Combined use of tractographyintegrated functional neuronavigation and direct fiber stimulation. J Neurosurg 102:664-672, 2005
18. Kawaguchi T, Kumabe T, Saito R, Kanamori M, Iwasaki M, Yamashita Y, Sonoda Y, Tominaga T: Practical surgical indicators to identify candidates for radical resection of insuloopercular gliomas. J Neurosurg 121:1124-1132, 2014

19. King RB, Schell GR: Cortical localization and monitoring during cerebral operations. J Neurosurg 67:210-219, 1987

20. Knauth M, Wirtz CR, Tronnier VM, Aras N, Kunze S, Sartor $\mathrm{K}$ : Intraoperative MR imaging increases the extent of tumor resection in patients with high-grade gliomas. AJNR Am J Neuroradiol 20:1642-1646, 1999

21. Kubben PL, ter Meulen KJ, Schijns OE, ter Laak-Poort MP, van Overbeeke $\mathrm{JJ}$, van Santbrink $\mathrm{H}$ : Intraoperative MRIguided resection of glioblastoma multiforme: A systematic review. Lancet Oncol 12:1062-1070, 2011

22. Kumabe T, Higano S, Takahashi S, Tominaga T: Ischemic complications associated with resection of opercular glioma. J Neurosurg 106:263-269, 2007

23. Lacroix M, Abi-Said D, Fourney DR, Gokaslan ZL, Shi W, DeMonte F, Lang FF, McCutcheon IE, Hassenbusch SJ, Holland E, Hess K, Michael C, Miller D, Sawaya R: A multivariate analysis of 416 patients with glioblastoma multiforme: Prognosis, extent of resection, and survival. J Neurosurg 95:190-198, 2001

24. Lang FF, Olansen NE, DeMonte F, Gokaslan ZL, Holland EC, Kalhorn C, Sawaya R: Surgical resection of intrinsic insular tumors: Complication avoidance. J Neurosurg 95:638-650, 2001

25. Lehéricy S, Duffau H, Cornu P, Capelle L, Pidoux B, Carpentier A, Auliac S, Clemenceau S, Sichez JP, Bitar A, Valery CA, Van Effenterre R, Faillot T, Srour A, Fohanno D, Philippon J, Le Bihan D, Marsault C: Correspondence between functional magnetic resonance imaging somatotopic and individual brain anatomy of the central region: Comparison with intraoperative stimulation in patients with brain tumors. J Neurosurg 92:589 598, 2000

26. Nimsky C, Fujita A, Ganslandt O, Von Keller B, Fahlbusch R. Fahlbusch R: Volumetric assessment of glioma removal by intraoperative high-field magnetic resonance imaging. Neurosurgery 55:358-370, 2004

27. Nimsky C, Ganslandt O, Tomandl B, Buchfelder M, Fahlbusch R: Low-field magnetic resonance imaging for intraoperative use in neurosurgery: A 5-year experience. Eur Radiol 12: 2690-2703, 2002

28. Nimsky C, Ganslandt O, Von Keller B, Romstöck J, Fahlbusch R: Intraoperative high-field-strength MR imaging: Implementation and experience in 200 patients. Radiology 233: $67-78,2004$

29. Nimsky C, von Keller B, Ganslandt O, Fahlbusch R: Intraoperative high-field magnetic resonance imaging in transsphenoidal surgery of hormonally inactive pituitary macroadenomas. Neurosurgery 59: 105-114, 2006

30. Ntoukas V, Krishnan R, Seifert V: The new generation polestar n20 for conventional neurosurgical operating rooms: $A$ preliminary report. Neurosurgery 62: 82-89, 2008

31. Ren H, Chen X, Sun G, Hu S, Zheng G, Li F, Li J, Xu $B$ : Resection of subependymal giant cell astrocytoma guided by intraoperative magnetic resonance imaging and neuronavigation. Childs Nerv Syst 29:1113-1121, 2013 
32. Roessler K, Donat M, Lanzenberger R, Novak K, Geissler A, Gartus A, Tahamtan AR, Milakara D, Czech T, Barth M, Knosp $\mathrm{E}$, Beisteiner R: Evaluation of preoperative high magnetic field motor functional MRI (3 Tesla) in glioma patients by navigated electrocortical stimulation and postoperative outcome. J Neurol Neurosurg Psychiatry 76:1152-1157, 2005

33. Ryken TC, Frankel B, Julien T, Olson JJ: Surgical management of newly diagnosed glioblastoma in adults: Role of cytoreductive surgery. J Neurooncol 89:271-286, 2008

34. Sanai N, Berger MS: Glioma extent of resection and its impact on patient outcome. Neurosurgery 62:753-764, 2008

35. Sanai N, Polley MY, Berger MS: Insular glioma resection: Assessment of patient morbidity, survival, and tumor progression. J Neurosurg 112:1-9, 2010

36. Sanai N, Polley MY, McDermott MW, Parsa AT, Berger MS: An extent of resection threshold for newly diagnosed glioblastomas. Clinical article. J Neurosurg 115:3-8, 2011

37. Senft C, Franz K, Blasel S, Oszvald A, Rathert J, Seifert V, Gasser T: Influence of iMRl-guidance on the extent of resection and survival of patients with glioblastoma multiforme. Technol Cancer Res Treat 9: 339-346, 2010

38. Shelley BP, Trimble MR: The insular lobe of Reil-its anatamicofunctional, behavioural and neuropsychiatric attributes in humans - a review. World J Biol Psychiatry 5:176-200, 2004

39. Simon M, Neuloh G, von Lehe M, Meyer B, Schramm J: Insular gliomas: The case for surgical management. Clinical article. J Neurosurg 110:685-695, 2009
40. Sun GC, Chen XL, Zhao Y, Wang F, Hou BK, Wang YB, Song ZJ,Wang D, Xu BN: Intraoperative high-field magnetic resonance imaging combined with fiber tract neuronavigationguided resection of cerebral lesions involving optic radiation. Neurosurgery 69:1070-1084, 2011

41. Sun GC, Chen $X L$, Zhao $Y$, Wang $F$, Song ZJ, Wang YB, Wang D, Xu BN: Intraoperative MRI with integrated functional neuronavigation-guided resection of supratentorial cavernous malformations in eloquent brain areas. J Clin Neurosci 18:1350-1354, 2011

42. Vanaclocha V, Sáiz-Sapena N, García-Casasola C: Surgical treatment of insular gliomas. Acta Neurochir 139:1126-1135, 1997

43. Wrensch M, Rice T, Miike R, McMillan A, Lamborn KR, Aldape $\mathrm{K}$, Prados MD: Diagnostic, treatment, and demographic factors influencing survival in a population-based study of adult glioma patients in the San Francisco Bay Area. Neuro Oncol 8:12-26, 2006

44. Yasargil MG, von Ammon K, Cavazos E, Doczi T, Reeves JD, Roth P: Tumours of the limbic and paralimbic systems. Acta Neurochir (Wien) 118:40-52, 1992

45. Zentner J, Meyer B, Stangl A, Schramm J: Intrinsic tumors of the insula: A prospective surgical study of 30 patients. $J$ Neurosurg 85:263-271, 1996

46. Zhao Y, Chen X, Wang F, Sun G, Wang Y, Song Z, Xu B: Integration of diffusion tensor-based arcuate fasciculus fibre navigation and intraoperative MRI into glioma surgery. J Clin Neurosci 19:255-261, 2012 